\title{
ON IMPLICIT FUNC'IIUNS AND THEIR DIFFERENTIALS
}

\author{
$B y$ W. H. Youna, Sc.D., F.R.S. \\ [Received January 20th, 1909.-Read February 11th, 1909.]
}

Introductory.

1. In the earlier treatises on the differential calculus, the transition from the consideration of explicit to that of implicit functions of a real variable was made almost without comment. The existence and differentiability of the latter class of functions was tacitly assumed as well as all necessary properties of the function of two variables which, when equated to zero, gives us the defining equation. Needless to add that the proof of the rules for obtaining the successive differential coefficients lacked clearness and rigour. In the more modern treatises, on the other hand, the transition is marked-is, indeed, abrupt in character. The reader is suddenly transported to a discussion of the properties of power series of one or more variables, so that instead of the attention being confined, as in the case of the differential coefficients of explicit functions, to a certain neighbourbood of the point in question, to what goes on, in fact, im Kleinen, he is obliged to consider the whole portion of space inside a certain circle or region of convergence, and to contemplate what goes on im Grossen. It is difficult to see how this mode of treatment can be justified from the point of view of the theory of functions of a real variable, or, indeed, to take another example, from that of differential geometry.

In the theory of functions of a real variable explicit functions not expressible by power series are an everyday occurrence, and there seems no reason for restricting the nature of implicit functions. In differential geometry, on the other hand, we are rarely concerned with a more thron limited number of differential coefficients, supposed to exist at the point under consideration, and the question whether the higher differential coefficients exist or not does not arise and has little interest.

The new definitions of differentials and the fundanental theorems concerning them given in my paper: "On Differentials," presented lately to the Society, systematise the discussion of implicit functions im Kleinen.

The results obtained in the present paper for implicit functions of any number of variables correspond precisely to the results for a single 
independent variable and include them as a special case, differentials taking the place of differential coefficients. The scope of the results corresponds exactly to the amount of assumption made in each case, and the ordinary rules of the differential calculus are shewn rigidly to apply for implicit functions defined in the wide sense of the present paper.

As far as I know, the only theorem on the subject of implicit functions not necessarily analytic previously known is the following one due to Dini, ${ }^{*}$ and its generalisation for several variables and more than one equation :-

If the equation $f(x, y)=0$ is satisfied by the values $x=a, y=b$, and if the function $f(x y)$ and its first differential coefficients at $(a, b)$ are continuous with respect to $(x, y)$, and if $f_{y}$ is not zero at the point $(a, b)$, then one, and only one, function $y$ of $x$ exists satisfying the equation $f(x, y)=0$ and having the value $b$ when $x$ is a. Moreover, this function possesses at $x=a$ a differential coefficient.

These theorems, which it will be noticed do not involve the idea of differential at all, form a very special case of those given in the present paper.

The theory takes its simplest form when the defining functions possess at least a second differential, the corresponding general theorem obtained being as follows:-

If $f_{1}(x, y), f_{2}(x, y), \ldots, f_{r}(x, y)$ are $r$ functions of the $m$ variables $x$ and the $r$ variables $y$, which are zero when the $x$ 's are equal to $a$ 's and the $y$ 's to b's, i.e., at the point $(a, b)$, and have n-th differentials there, where $n \geqslant 2$, and if the Jucobian $J$ of the f's with respect to the $y$ 's is not zero at the point $(a, b)$, then there exist unique functions $y_{1}, y_{2}, \ldots, y_{\text {. }}$ of the $x$ 's, which have the values $b_{1}, \ldots, b_{r}$ at the point a, and, throughout a closed neighbourhood of that point, make all the f's identically zero. Moreover, these functions $y$ have at the point a n-th differentials whose values may be obtained from the usual equations. (Theorem 9, §14.)

When the defining function or functions have only first differentials, the theorem takes a much less simple form. Taking, for simplicity, the case of two variables $x$ and $y$ and a single defining function $f(x, y)$, the theorem is as follows :-

If $f(x, y)$ be a function of $x$ and $y$, whose value at the point $(a, b)$ is zero, and which in a certain closed neighbourhood of the point $(a, b)$ is continuous with respect to $x$ and with respect to $y$, and possesses at 
the same point a finite differential coefficient with respect to $y, f_{b}$, different from zero, then a function $y$ of $x$ exists with the following properties :-

(1) Its value is $b$ when $x$ is $a$.

(2) When substituted in $f(x, y)$ it makes $f^{\prime}(x, y)$ zero thioughout a certain neighbourhood of the point $(a, b)$.

(3) Further, if, for each fixell value of $x, f$ is a monotone never constant function of $y$, this function $y$ of $x$ is unique.

(4) Further, if $f$ is a continuous function of the ensemble $(x, y)$, this function $y$ is a continuous function of $x$.

(5) Finally, if $f$ possesses at the point $(a, b)$ a first differential, then this function $y$ possesses at the point $x=a$ a first differential coefficient.

This enunciation seems, perhaps, unduly complicated, but examples are given shewing that no simpler statement will serve.

The paper falls into three parts : in the first part certain preliminary theorems about differentials, required in the sequel, are proved; in Part II., implicit functions defined by a single equation, and, in Part III., those defined by two or more equations are discussed.

\section{Part I.}

\section{$\$ \S 2-5$. Preliminary Theorems.}

2. Theorem 1.-If $u$ be a function of the $r$ variables $x_{1}, x_{2}, \ldots, x_{r}$ and the $s$ variables $y_{1}, y_{2}, \ldots, y_{s}$, while $v$ is a function of the $s$ variables $y_{1}, y_{2}, \ldots, y_{s}$ and the $t$ variables $z_{1}, z_{2}, \ldots, z_{t}$, and, if $u$ possess an $n$-th differential with respect to the ensemble of the $x$ 's and the $y$ 's at the point $\left(a_{1}, a_{2}, \ldots, a_{r} ; b_{1}, b_{2}, \ldots, b_{s}\right)$, and $v$ possess an $n$-th differential with respect to the ensemble of the $y$ 's and the $z$ 's at the point $\left(b_{1}, b_{2}, \ldots, b_{s}\right.$; $\left.c_{1}, c_{2}, \ldots, c_{t}\right)$, then $u+v$ and $u-v$ both possess $n$-th differentials with respect to the ensemble of the $x$ 's, $y$ 's, and $z^{\prime}$ 's at the point

$$
\left(a_{1}, a_{2}, \ldots, a_{r} ; b_{1}, b_{2}, \ldots, b_{s} ; c_{1}, c_{2}, \ldots, c_{t}\right) .
$$

The theorem is obvious when $n=1$, and follows by induction for $n>1$, from the very mode in which we have defined the $n$-th differential at a point as the first differential of the $(n-1)$-th differential, supposed to exist at the point and in the neighbourhood.

[N.B.--The $y$ 's and b's may, of course, in a particular case, be absent; or, again, the $x$ 's and $z$ 's with the $a$ 's and $c$ 's may be absent.]

3. Thronem 2.-Under the same hypotheses, the product uv possesses an n-th differential at the point in question. 
To prove the theorem when $n=1$ we write, for shortness, $x$ for the ensemble of the $x$ 's, $y$ for that of the $y$ 's, and $z$ for that of the $z$ 's, and $D$ for the operator

$$
\Sigma\left(h_{i} \frac{d}{d x_{i}}+k_{i^{\prime}} \frac{d}{d y_{i^{\prime}}}+l_{i^{\prime \prime}} \frac{d}{d z_{i^{\prime \prime}}}\right) .
$$

Writing $u=f(x, y), \quad v=g(y, z), \quad u v=F(x, y, z)$,

we then have, using the rule for differentiating a product,

Now

$$
D F=f D g+g D f \text {. }
$$

$$
\begin{aligned}
F(x+h, y+k, z+l) & =f(x+h, y+k) g(y+k, z+l) \\
= & \left\{f(x, y)+D f(x, y)+E_{1}\right\}\left\{g(y, z)+D g(y, z)+E_{2}\right\},
\end{aligned}
$$

when $f$ and $g$ both have first differentials at the point $(x, y, z)$ in question. Here $E_{1}$ is a linear function of the $h$ 's and $k$ 's and $E_{2}$ a linear function of the $k$ 's and $l$ s, whose coefficients have zero as limit when the $l$ 's, $k$ 's, and $l$ 's approach zero in any manner.

Hence, multiplying out the right-hand side of (2) and using (1),

$$
F(x+l, y+k, z+l)=F(x, y, z)+D F+E_{9},
$$

where $E_{3}$ is a linear function of the $h^{\prime} \mathrm{s}, k^{\prime} \mathrm{s}$, and $l$ 's whose coefficients have zero as limit when the $h$ 's, $k$ 's, and $l$ 's approach zero in any manner.

The equation (3) shows that, by the definition of a first differential, $F$ has a first differential at the point in question, and it is given by

$$
\Delta F=f \Delta g+g \Delta f,
$$

since the operator $D$, which gives the differential $\Delta$, if it exists, obeys the relation (1).

This proves the theorem when $n=1$. Moreover, it shews that the differential of the product is a linear function of $u, v$ and their differentials, and therefore, by Theorem 1 and the present theorem for $n=1$, has itself a first differential with respect to the ensemble $(x, y, z)$ at the point in question, if $u, v$ and their first differentials have first differentials at the corresponding points. In other words, recalling the definition of a second differential as the differential, if it exists, of the first differential, supposed to exist at the point and in the neighbourhood, the product $u v$ has a second differential with respect to the ensemble $(x, y, z)$ at the point $(a, b, c)$, provided $u$ and $v$ have second differentials at the points $(a, b)$ and $(b, c)$ respectively.

This proves the theorem when $n=2$. Noreover, it shows that the 
second differential of the product is a linear function of $1, r$ and their first and second differentials, viz.,

$$
\Delta^{2} F=\Delta(f \Delta g)+\Delta(g \Delta f)=f \Delta^{2} g+2 \Delta f \Delta g+g \Delta^{2} f .
$$

Hence, by similar ressoning, the theorem follows for $n=3$, and so generally, by induction, the form of the $n$-th differential of the product being identical with Leibniz's formula for the $n$-th differential coefticient of a product.

4. Thensem 3.-Under the same hypotheses, if $x$ is defined by the identity

$$
u+v w=0
$$

wo has an $n$-th differential at the point $(a, b, c)$ provided $v$ does not ranish at the point $(b, c)$.

To prove the theorem for $n=1$, we have, using $F(x, y, z)$ for $w$,

$$
f(x+h, y+k)+g(y+k, z+l) F(x+h, y+k, z+l)=0 ;
$$

or, since $f$ and $g$ have first differentials at the point $(x, y, z)$ in question, $f(x, y)+D f(x, y)+E_{1}+\vdots g(y, z)+D g(y, z)+E_{2} ; F(x+h, y+k, z+l)=0$.

Multiplying this equation by $g(y, z)$ and subtracting (1), after multiplying it by the coefficient of $F(x+h, y+k, z+l)$ in (3), we get

$$
\begin{aligned}
& \left.-f(x, y) \vdots D g(y, z)+E_{2}\right\}+g(y, z)\left\{D f(x, y)+E_{1}\right\} \\
& \quad+g(y, z) \vdots g(y, z)+D g(y, z)+E_{2}: \vdots F(x+h, y+h, z+l)-F(x, y, z) !=0,
\end{aligned}
$$

that is,

$$
\begin{aligned}
g(y, z) D f(x, y) & -f(x, y) D g(y, z)+E_{3} \\
& +i g(y, z){ }^{2}: F(x+h, y+k, z+l)-F(x, y, z) ;=0,
\end{aligned}
$$

where

$$
\begin{aligned}
E_{3}=E_{1} g(y, z)-E_{2} f(x, y) & \\
& +g(y, z) \vdots D g(y, z)+E_{2}: \vdots F(x+h, y+k, z+l)-F(x, y, z) \vdots,
\end{aligned}
$$

so that $E_{3}$ is a linear function of $h, k$, and $l$ whose coeflicients have zero as limit when $h, k$, and $l$ approach zero in any manner whatever.

Now, if $g$ does not vanish at the point in question, we luity divide (4) by $g^{2}$, whence, using the expressions for the differential coefticient of a product or proving those expressions independently from the equation Bgk. 2. voL. 7 . No. 1030. 
(4), we get

$$
F(x+h, y+k, z+l)=F(x, y, z)+D F(x, y, z)+E_{3} / g^{2},
$$

where

$$
D F(x, y, z)=\frac{g D f-f D g}{g^{2}} .
$$

This shews that $F$ has a first differential at the point whose form is, of course, precisely that of the differential coefficient of a quotient. This proves the theorem for $n=1$.

If $u$ and $v$ have second differentials at the point, and therefore first differentials in the neighbourhood, we can, since $g$ is continuous, so restrict the neighbourhood that $g$ is never zero in it. The equations (5) and (6) will then hold throughout the neighbourhood, so that $w$, that is $F$, will have a first differential at every point of the neighbourhood given by the equation

$$
\{g(y, z) \Delta f(x, y)-f(x, y) \Delta g(y, z)\}-\{g(y, z)\}^{2} \Delta F(x, y, z)=0,
$$

which is an equation of the original form (1), in which the place of $u$ is taken by the difference of products of functions which are known to have first differentials, that is, by a function which has a first differential at the point in question, and the place of $u$ is taken by $-u^{2}$, that is $-g^{2}$, which again, by Theorem (2), has a first differential at the point and is not zero there. Hence, applying the theorem already proved for $n=1$, it follows that $F$ has a second differential, and that this is again given by an equation of the form (1).

Using induction, it easily follows that the theorem is true for all values of $n$, and that the successive differentials may be obtained by the rule for finding the successive differential coefficients of a quotient.

5. TheoReM 4.-If $f\left(x_{1}, x_{2}, \ldots, x_{p} ; z_{1}, z_{2}, \ldots, z_{n}\right)$ be a function of the $(p+m)$ variables $x$ and $z$ and have a first differential at the point $\left(a_{1}, a_{2}, \ldots, a_{p} ; c_{1}, c_{2}, \ldots, c_{m}\right)$, and if the $z$ 's are functions of certain of the $x$ 's and of $s$ other variables $y_{1}, y_{2}, \ldots, y_{s}$ and have the values $c_{1}, c_{2}, \ldots, c_{m}$ when the $x$ 's have the values $a_{1}, a_{2}, \ldots$ and the $y$ 's the values $b_{1}, b_{2}, \ldots, b_{s}$, and, if at the point $\left(a_{1}, a_{2}, \ldots ; b_{1}, b_{2}, \ldots\right)$ each of the $z$ 's has an $n$-th differential with respect to the variables on which it depends, then $f$, regarded as a function of all the $p$ variables $x$ and the $m$ variables $y$, has an $n$-th differential at the point $\left(a_{1}, a_{1}, \ldots, a_{p} ; b_{1}, b_{2}, \ldots, b_{m}\right)$.

It is first to be remarked that we may suppose each variable $z$ to be a function of all the $p+m$ variables $x$ and $y$. For a function of some of these variables is only a special case of a function of all of them, and its differential coefficient with respect to any missing variable is zero, so 
that its differential may be regarded as its differential with respect to all the variables.

Having premised this, it is unnecessary explicitly to mention the $x$ 's directly involved in $f$, since any such $x$ is then a special case of a function of the $(p+m)$ variables, and may itself be denoted by the letter $z$. Having done this, there is no longer any reason for distinguishing between the $y$ 's and the $x$ 's, and we may replace $f\left(x_{1}, x_{2}, \ldots, x_{p} ; z_{1}, z_{2}, \ldots, z_{m}\right)$ by $f\left(z_{1}, z_{2}, \ldots, z_{m}\right)$, where the $z$ 's are functions of $x_{1}, x_{2}, \ldots, x_{p}$.

To prove the theorem for $n=1$, let us write

$$
f\left(z_{1}, \ldots, z_{m}\right)=F\left(x_{1}, \ldots, x_{p}\right)
$$

or, shortly, using $x$ and $z$ for the ensembles of the $x$ 's and the $z$ 's respectively

$$
f(z)=F(x) \text {. }
$$

Since the $z$ 's have first differentials at the point considered,

$$
z_{i}=c_{i}+D z_{i}+E_{i},
$$

where $E_{i}$ is a linear function of $h_{1}, h_{2}, \ldots, h_{n}$ whose coefficients have zero as limit when the $h$ 's approach zero in any manner, and $D$ denotes the operator

$$
h_{1} \frac{d}{d x_{1}}+h_{2} \frac{d}{d x_{2}}+\ldots+h_{p} \frac{d}{d x_{p}} .
$$

Hence

$$
F(x)=f\left\{c_{1}+D_{1} z_{1}+E_{1}, \ldots, c_{m}+D z_{m}+E_{m}\right\}^{\prime}
$$

or, since $F$ has a first differential,

$$
F(x)=f(c)+\sum_{:}(D z+E) \frac{d f}{d z}+E^{\prime},
$$

where $E^{\prime}$ is a linear function of the increments $D z+E$, and therefore of the $p$ variables $h$, whose coefficients have zero as limit.

Hence, keeping all the variables except one constant and proceeding to the limit after dividing by the increment of that variable, we get

and therefore

$$
\frac{d}{d x_{i}}\{F(x)\}=\sum_{\Sigma} \frac{d f}{d z} \frac{d z}{d x_{i}},
$$

Hence, by (1),

$$
D F(x)=\sum_{\Sigma}\left(D z \frac{d f}{d z}\right) .
$$

where $E^{\prime \prime}$ is again a linear function of the $h$ 's whose coefficients have zero as limit. That is, $F(x)$ has a first differential at the point considered.

This proves the equation for $n=1$.

If $n=2$, the above reasoning shews that the first differential of $F$, which, since it exists, has the form $D F$ and is therefore given by the 
equation (2), has a first differential, and therefore $F$ has a second differential with respect to the $x$ 's. For $D F$ is, by (2), expressed as a sum of terms of the form $D z(d f / d z)$ consisting of two factors : the first a function of the $x$ 's having a first differential with respect to the $x$ 's at the point considered, the second a function of the $z$ 's having a first differential with respect to the $z$ 's at the corresponding point. Each such term has, therefore, by Theorem 2, a first differential with respect to the $x$ 's and $z$ 's, and therefore, by what has been proved, a first differential at the point considered with respect to the $x$ 's alone. Hence the sum of all the terms has the same property.

This proves the theorem for $n=2$.

The theorem now follows, similarly, by induction, assuming that it holds for $n=r$, and that the $r$-th differential with respect to $x$ is a function of the $x$ 's and $z$ 's which has a first differential with respect to all the $x$ 's and $z^{\prime}$ 's which it involves.

\section{Part II.}

$\$ \$ 6-11$. Existence and Differentials of an Implicit Function defined by a Single Equation.

6. Theores 5.-If $f(x, y)$ be a function of $x$ and $y$, whose value at the point $(a, b)$ is zero, and which in a certain closed neighbourhood of the point $(a, b)$ is continuous with respect to $x$ and with respect to $y$, and possesses at the same point a finite differential coefficient with respect to $y, f_{b}$ different from zero, then a function $y$ of $x$ exists with the following properties :-

(1) Its value is $b$ when $x$ is a.

(2) When substituted in $f(x, y)$ it makes $f(x, y)$ zero throughout a certain neighbourhood of the point $(a, b)$.

(3) Further, if, for each fixed value of $x, f$ is throughout some closed neighbourhood of the point $(a, b)$ a monotone never constant function of $y$, this function $y$ of $x$ is unique.

(4) Further, if $f$ is a continuous function of the ensemble $(x, y)$ in the closed neighbourhood of the point $(a, b)$, this function $y$ of $x$ is a continuous function of $x$.

(5) Finally, if $f$ possesses at the point $(a, b)$ a first differeniial, then this function $y$ of $x$ possesses at the point $x=a$ a first differeniial 
coefficient, whose value $p$ is given by

$$
f_{a}+p f_{b}=0 \text {. }
$$

For definiteness it will be assumed that $f_{b}$ is positive. It then follows from the definition of a differential coefficient, and the fact that $f(a, b)$ is zero, that we can find a stretch on the ordinate of the point $(a, b)$, having that point as centre. such that in the upper half $f$ is greater than zero, and in the lower half it is less than zero. Hence also, since $f$ is continuous with respect to $x$, we can draw two stretches* parallel to the axis of $x$, having the end-points of the stretch just found for centres, and of equal length, such that in the upper one $f$ is greater than zero, and in the lower one $f$ is less than zero.

Completing this rectanglet we obtain a closed neighbourhood of the point $(a, b)$, such that in it on each ordinate $f$ has a positive value and a negative value, and therefore, since $f$ is continuous with respect to $y$, assumes the value zero at one or more points forming a closed set.

The $y$-coordinate of the lowest of these points on each ordinate constitutes a function $y$ of $x$ having the properties (1) and (2).

If $f$ is for each fixed value of $x$, a monotone never constant function of $y$, this function $y$ of $x$ is unique, for $f$ then only assumes each of its values once on each ordinate, in particular $f$ is zero once only. This proves (3).

To prove (4), we only have to notice that, if $f$ is continuous with respect to the ensemble $(x, y)$, the plane $\ddagger$ set of all its zeros in the closed neighbourhood chosen forms a closed set. Hence, taking any sequence $x_{1}, x_{2}, \ldots$ having $x$ as limit, the corresponding zeros have as limit the zero on the limiting ordinate, so that the function $y$ of $x$ has for every value of $x$ a value equal to the unique limit of values in the neighbourhood, i.e., it is a continuous function of $x$.

Finally, if $f$ has a first differential at the point $(a, b)$, there is a closed neighbourhood of the point such that throughout it

$$
f(a+h, b+k)=h\left(f_{a}+e_{1}\right)+k\left(f_{b}+e_{2}\right),
$$

where the $e$ 's have zero as limit when $h$ and $k$ approach zero in any manner. Hence, inserting for $b+k$ our function $y$,

$$
0=(x-a)\left(f_{a}+e_{1}\right)+(y-b)\left(f_{b}+e_{2}\right),
$$

- In the corresponding $(n+1)$-dimensional discussion these :ure what may be called hyperstretches : thus for three dimensions they are squares, for four dimensions cubes, and so on.

$\dagger(n+1)$-dimensional parallelepiped.

$\ddagger(n+1)$-dimensional. 
so that

$$
\frac{y-b}{x-a}=-\frac{f_{a}+e_{1}}{f_{b}+e_{2}}
$$

where, when $x$ approaches $a$ in any manner, $y$ being continuous has the limit $b$, and therefore $e_{1}$ and $e_{2}$ both have the limit zero.

Proceeding to the limit, we have, by the definition of a differential coefficient,

that is,

$$
\frac{d y}{d x}=-f_{a} / f_{b}
$$

Note.-If the condition which secures the uniqueness be omitted it is evident that the uppermost of all the functions $y$ of $x$ which make $f=0$ is upper-semi-continuous, and that the lowest is lower-semi-continuous.

Cor. 1.-We may replace the condition that throughout the neighbourhood $f$ should be for each fixed value of $x$ a monotone nowhere constant function of $y$, by the condition that $f_{y}$ should exist throughout a closed neighbourhood of $(a, b)$ and be nowhere zero.

In fact, if $f_{y}$ is nowhere zero in the neighbourhood it has always the same sign on each ordinate, since for each fixed value of $x$ it assumes on the corresponding ordinate every value between its upper and lower bounds, and therefore could not have opposite signs without being somewhere zero.

It is for the rest clear by applying the Theorem of the Mean that, if $f$ vanishes at two points on an ordinate $f_{y}$ must vanish at some point between the two points, so that the condition in question necessarily excludes this possibility.

Cor. 2.-We may replace the condition in question by the following :*that $f_{y}$ should exist throughout a closed neighbourhood of the point $(a, b)$, and be continuous at that point with respect to the ensemble $(x, y)$.

For in this case we can assign a closed neighbourhood of the point $(a, b)$ throughout which $f_{y}$ has the same sign as at $(a, b)$, and therefore never vanishes, so that we can apply Cor. 1.

7. The following examples shew that the theorem just proved contains the utmost that can be stated under the circumstances there enunciated.

* A particular case of this is Dini's theorem which replaces this condition by requiring the continuity of both $f_{x}$ and $f_{y}$ at the point $(a, b)$. C.p. Dini's lithographed lectures. 


$$
\text { Ex. 1.-Let } \quad f(x, y)=y-x-x^{2}-\frac{2 x^{4}}{y-x},
$$

except in that portion of the plane bounded by the curves

$$
y=x+2 x^{2}, \text { and } y=x-x^{2},
$$

in which the line $y=x$ lies, in which portion

$$
f(x, y)=0 .
$$

Here, when $x=0, f=y$, therefore ut the origin $d f / d y$ exists, and is unity. When $y=0, f=-x-x^{2}+2 x^{3}$, and therefore $d f / d x$ exists at the origin and is -1 .

Evidently $f$ is a continuous function of $(x, y)$ throughout the plane, vanishing as it does on the boundary of the two portious of space in which it has values given by (1) and (2) respectively.

Evidently ulong any one of the curves

$$
y=x+\frac{2-c}{1+c} x^{2},
$$

where $c$ has any positive value, rational or irrational, $f$ vanishes. Thus there is a more than countably infinite number of continuous functions of $x$ which make $f$ zero.

In spite, however, of this fact, $f$ possesses a first differential at the origin. For suppose $e$ any assigned small positive quantity. Then we can shew that throughout the square whose centre is the origin, and sides parallel to the coordinate axes, and of length $\frac{1}{2} e$, we have the following equation

$$
f(x, y)=x\left(\frac{d f}{d x}\right)_{0,0}+y\left(\frac{d f}{d y}\right)_{0,0}+e_{1} x,
$$

where $e_{1}$ is numerically less than $e$.

In fact, if $(x, y)$ be any point in the portion of the plane in which (1) holds, we have

$$
y-x \geqslant 2 x^{2} \text { or } \leqslant-x^{2} \text {; }
$$

and therefore numerically $\geqslant x^{2}$. Hence $2 x^{4} /(y-x)$ is numerically $<3 x^{2}$. Hence

$$
f(x, y)=y-x+k x^{2},
$$

where $k$ is numerically less than 4 , and $k x$ accordingly numerically less than $e$, since the point in question is supposed chosen in the square previously referred to.

Again, if $(x, y)$ lie in the portion of space in which (2) holds,

$$
y-x \leqslant 2 x^{2} \text { and } \geqslant-x^{2} ;
$$


and is therefore numerically less than $3 x^{2}$. Hence

$$
f(x, y)=0=y-x-(y-x)=y-x+k^{\prime} x^{2},
$$

where $k^{\prime}$ is numerically less than 3 , and $k^{\prime} x$ accordingly numerically less than $e$. From (3) and (t), bearing in mind the values of $d f / d x$ and $d f / d y$ at the origin the required result follows.

$E x$. 2.- Let $f$ have the same value as before, except in the portion of the plane between the curves

$$
y=x+2 x^{2}, \quad y=x-x^{2} .
$$

In this portion of the plane let

$$
\dot{f}(x, y)=\left(x+2 x^{2}-y\right)\left(x-x^{2}-y\right) .
$$

$A$ s before, at the origin $d f / d y=1$, and $d f / d x=-1$, and we prove by in argument similar to that used in the preceding case that $f$ possesses a differential.

Here, on the other hand, there are two, and only two, functions of $y$ which, when substituted for $y$ in $f$, make $f$ vanish, these functions being both of them continuous.

8. THEовем 6.-If $f(x, y)$ is a function of the ensemble $(x, y)$ which is zero at $(a, b)$, and possesses there an n-th differential, where $n$ is greater than unity, then, provided $d f / d y$ is not zero at the point $(a, b)$, we can find a closed neighbourhood of the point $(a, b)$, in which there is one, and only one, function $g(x)$ of $x$ which has the value $b$ when $x=a$ and when substituted for $y$ makes $f(x, y)$ identically zero. Further, this function possesses an n-th differential coefficient at the point $(a, b)$, which may be obtained by equating to zero the total successive differential coefficients of the finction $f^{\prime}(x, y)$, obtained by the ordinary rule.

First, to prove the theorem when $n=2$, we remark that, since the function bas a second differential at the point $(a, b)$, a first differential exists at and in a closed neighbourhood of the point $(a, b)$. Also since $f$ has a second differential at the point $(a, b), f_{y}$ has a first differential there, and is therefore continuous at $(a, b)$. Hence, remembering that $f_{b}$ is different from zero, we may so choose our neighbourhood that $f_{y}$ is different from zero at every point considered. The neighbourhood so chosen is then such that the conditions of Theorem 5 are satisfied at every point. Hence corresponding to each point $x$ there is a "tile," that is, a clused rectangle with $x$ us centre, and in this tile a unique function $g(x)$, 
such that $f[x, g(x)]$ is identically zero throughout the tile, and further

$$
f_{x}+g^{\prime}(x) f_{y}=0,
$$

or, symbolically, $\quad\left(\frac{d}{d x}+y^{\prime} \frac{d}{d y}\right) f(x y)=0$,

where, after differentiating, we have to insert $g(x)$ for $y$.

These tiles overlap, but, since in each tile the function $g(x)$ is unique, it follows that the value of $g(x)$ is independent of the particular tile used in determining its value, and is the same whether or no that tile was the one with $x$ as centre. Thus we have a unique function $g(x)$ defined throughout the whole neighbourhood, and it has a differential coefficient at each point, given by the identical equation (1).

Now, since $f$ has a second differential at the point $(a, b), f_{x}$ and $f_{y}$ both have first differentials there, and therefore have total differential coefficients with respect to $x$ when we replace $y$ by $g(x)$. Also the right-hand, and therefore the left-hand side of (1) has a total differential coefficient, whose value is zero. Thus, since $f_{y}(x)$ is not zero at $(a, b)$, we may apply Lemma 2, and say that $g^{\prime}(x)$ has a first differential coefficient at the point $(a, b)$, and that it is given by totally differentiating the identity (1) with respect to $x$, and putting $x=a, y=b$. That is, $g(x)$ possesses a second differential coefficient, and it is given by

$$
f_{a a}+2 f_{a b} g^{\prime}(a)+f_{b b}\left\{g^{\prime}(a)\right\}^{2}+f_{b}^{\prime} g^{\prime \prime}(a)=0,
$$

or, say, symbolically

$$
\left(\frac{d}{d x}+g^{\prime} \frac{d}{d y}\right)^{2} f(x, y)=0 \quad(x=a, y=b),
$$

that is, it is obtained by equating to zero the total differential coefficient of the second order of $f(x, y)$ with respect to $x$, when $y=g(x)$.

It will be noticed that we have, in performing the total differentiation, made no distinction between $f_{a b}$ and $f_{b a}$, in accordance with the results proved in my paper "On Differentials."

This proves the theorem when $n=2$.

Again, if $n=3$, not only, as we saw, is $f_{y}$ different from zero at each point of the neighbourhood of $(a, b)$, but at each such point $f$ has a second differential, so that the above reasoning applies, and we may assert that at each such point $g(x)$ has a second differential given by

$$
\left(\frac{d}{d x}+g^{\prime} \frac{d}{d y}\right)^{2} f(x, y)=0,
$$

that is, by

$$
\left(f_{x:}, \ldots\right)\left(1, g^{\prime}\right)^{2}+f_{y} g^{\prime \prime}(x)=0 \text {. }
$$


The reasoning by which we now deduce the existence and value of $g^{\prime \prime \prime}(a)$ from (2), is precisely similar to that by which we deduced the existence and value of $g^{\prime \prime}(a)$ from (1) in the case when $n=2$.

For, since $f$ has a third differential at $(a, b), f_{x x}, f_{x y}$ and $f_{y y}$ have first differentials there. Also $g^{\prime}(x)$ has a first differential coefficient, and therefore a first differential at the same point. Since the product and the sum of functions having a first differential at $(a, b)$ is a function having a first differential there, it follows that the quadratic $\left(f_{x x}, \ldots\right)\left(1, g^{\prime}\right)^{2}$ has a first differential at $(a, b)$. Moreover $f_{y}$ has a first differential at the same point. Hence both these functions $\left.\left(f_{x x}, \ldots\right\} 1, g^{\prime}\right)^{2}$, and $f_{y}$ have total differential coefficients with respect to $x$, when we replace $y$ by $g(x)$. Again the righthand side, and therefore the left-hand side of (2) has the total differential coefficient zero. Thus, since $f_{b}$ is different from zero, we may apply the Lemma, and say that $g^{\prime \prime}(x)$ has a first differential coefficient at the point $(a, b)$, and that it is given by totally differentiating the identity (2) with respect to $x$, and putting $x=a, y=b$. That is, $g^{\prime \prime \prime}(a)$ exists, and is given by

$$
\left(f_{\text {caaa }}, \ldots \backslash 1, g^{\prime}\right)^{3}+3\left\{f_{a b}+g^{\prime}(a) f_{b b}\right\} g^{\prime \prime}+f_{b} g^{\prime \prime \prime}=0,
$$

or, symbolically, by

$$
\left(\frac{d}{d x}+g^{\prime} \frac{d}{d y}\right) f(x, y)=0 \quad(x=a, y=b) .
$$

This proves the theorem for $n=3$. We have now only to notice that, if we have proved the theorem for $n=r$, it follows by corresponding reasoning that it is true for $n=r+1$. Hence, by induction, the truth of the theorem follows.

9. The equations giring in order the successive differential coeflicients, when written out at length are as follows :-

(1) $\left(\frac{d}{d x}+y^{\prime} \frac{d}{d y}\right) f=0, \quad$ or $\quad f_{x}+y^{\prime} f_{y}=0$;

(2) $\left(\frac{d}{d x}+y^{\prime} \frac{d}{d y}\right)^{2} f=0, \quad$ or $\quad\left(f_{x x}, f_{x y}, f_{y y} \gamma\left(1, y^{\prime}\right)^{2}+f_{y} y^{\prime \prime}=0\right.$;

(3) $\left(f_{x x x}, \ldots\right)\left(1, y^{\prime}\right)^{3}+3\left(f_{x y}+y^{\prime} f_{y y}\right) y^{\prime \prime}+f_{y} y^{\prime \prime \prime}=0$;

(4) $\left(f_{x x x x}, \ldots\right)\left(1, y^{\prime}\right)^{4}+6\left(f_{x x y}, \ldots\right)\left(1, y^{\prime}\right)^{2} y^{\prime \prime}+3 f_{y y} y^{\prime \prime 2}$

$$
+4\left(f_{x, y}+y^{\prime} f_{y y}\right) y^{\prime \prime \prime}+f_{y} y^{(4)}=0 ;
$$


(5) $\left(\frac{d^{5} f}{d x^{5}}, \ldots\right)\left(1, y^{\prime}\right)^{5}+\frac{5.4}{1.2}\left(f_{x x x y y}, \ldots\right)\left(1, y^{\prime}\right)^{8} y^{\prime \prime}+\frac{5.4 .3}{1.2 .3}\left(f_{x x y y}, \ldots\right)\left(1, y^{\prime}\right)^{2} y^{\prime \prime \prime}$

$$
+5\left(f_{x y}, \ldots \chi 1, y^{\prime}\right) y^{(4)}+15\left(f_{x y y}, \ldots \chi 1, y^{\prime}\right) y^{\prime 2}+10 f_{y ! !} y^{\prime \prime} y^{\prime \prime \prime}+f_{y} y^{(5)}=0 \text {, }
$$

and so on.

It should be noticed that it follows from the mode of formation of these equations that the coefficients are partial differential coefticients of $f$ such that, if any coefficient involves $r$ differentiations with respect to $y$, it is multiplied by precisely $r$ differential coefficients of $y$.

10. If in Theorem 5 we interpret the symbol $x$ to mean the ensemble $\left(x_{1}, x_{2}, \ldots, x_{n}\right)$, so that $f(x, y)$ means $f\left(x_{1}, x_{2}, \ldots, x_{n}, y\right)$, the theorem becomes a theorem in the theory of functions of $(n+1)$-variables, no alteration in the wording being required except in (5), which should now read as follows:-

(5) Finally, if $f$ possesses at the point $(a, b)$ a first differential, then this function $y$ of $x$ possesses at the point $x=a$ a first differential, whose value $\Delta y$ is given by the symbolic equation

$$
\left(h \frac{d}{d x}+\Delta y \frac{d}{d y}\right) f=0
$$

(that is, written out in full,

$$
h_{1} f_{x_{1}}+h_{2} f_{x_{3}}+\ldots+h_{n} f_{x_{n}}+\Delta y f_{y_{1}}=0 \text { ), }
$$

in which the x's are to be replaced by $a$ 's and $y$ by $b$.

The proof of this theorem is almost word for word the same as before; the insignificant verbal alterations have been already given in footnotes. For complete clearness the part of the proof which refers to (5) is given in full below.

"Finally, if $f$ has a first differential at the point $(a, b)$, there is a closed neighbourhood of the point such that throughout it

$$
f(a+h, b+k)=h\left(f_{a}+e\right)+k\left(f_{b}+e^{\prime}\right),
$$

that is, when written out fully,

$$
f\left(a_{1}+h_{1}, \ldots, a_{n}+h_{n}, b+k\right)=h_{1}\left(f_{a_{1}}+e_{1}\right)+\ldots+h_{n}\left(f_{a_{n}}+e_{n}\right)+k\left(f_{b}+e^{\prime}\right),
$$

where the $e$ 's have the limit zero when the $h$ 's and $k$ 's approach zero in any manner.

Hence, inserting for $b+k$ our function $y$,

$$
0=(x-a)\left(f_{a}+e\right)+(y-b)\left(f_{b}+e^{\prime}\right),
$$


so that $y=b+(x-a)\left(-\frac{f_{a}+e}{f_{b}+e^{\prime}}\right)=b+(x-a)\left(-\frac{f_{a}}{f_{b}}+\epsilon\right)$,

where, when $x$ approaches $a$ in any manner, $y$ being continuous, has the limit $b$; and therefore the $e$ 's all have the limit zero, and hence the same is true of the $\epsilon$ 's. Taking all the $x$ 's except one to have the corresponding values $a$, and then proceeding to the limit with the remaining $x$, after dividing the equation by $x-a$, we see that the corresponding $d y / d x$ exists at the point $a$, and has the value $-f_{a} / f_{b}$.

Hence the preceding equation proves that $y$ has at the point $a$ a first differential which is given by the equation

$$
\Delta y=(x-a)\left(-f_{a} / f_{b}\right)
$$

or, at length,

$$
\Delta y=h_{1}\left(-f_{a_{1}} \mid f_{b}\right)+\ldots+h_{n}\left(-f_{t_{1 n}} / f_{b}\right) \text {, }
$$

that is,

$$
\Delta y f_{b}+h_{1} f_{a_{1}}+\ldots+h_{n} f_{a_{n}}=0
$$

or, as we may write it, symbolically,

$$
\left(h_{1} \frac{\hat{\partial}}{\partial x_{1}}+\ldots+h_{n} \frac{\partial}{\partial x_{n}}+\Delta y \frac{\hat{c}}{\partial y}\right) f=0,
$$

or, shortly,

$$
\left(h \frac{\hat{c}}{\partial x}+\Delta y \frac{\partial}{\partial y}\right) f=0
$$

in which symbolic equation we are to understand that the values of $x$ and $y$ are after differentiation to be changed to $a$ and $b . "$

11. Theorem 6 may similarly be interpreted in space of $(n+1)$ dimensions. We merely have to change "differential coefficient" in the enunciation into "differential." It is unnecessary to reproduce the proof, but it may be well to give the equations determining the successive differentials more at length.

Corresponding to the equation (1) of $\S 8$, we now have

$$
\left(h \frac{d}{d x}+\Delta y \frac{d}{d y}\right) f=0,
$$

holding at every point of the closed neighbourhood, where, after differentiation, we have to insert $g(x)$ for $y$, and $\Delta y$ denotes the differential of this function of the $x$ 's at the point $x$, that is $\left(x_{1}, x_{2}, \ldots, x_{n}\right)$.

Thus $\Delta y$ does not involve $y$, and if we operate upon it with the operator $\left(h \frac{d}{d x}+\Delta y \frac{d}{d y}\right)$, we get the second differential $\Delta^{2} y$ if this exists. Now this operator $\left(h \frac{d}{d x}+\Delta y \frac{d}{d y}\right)$ is the operator which gives the total 
differential, when it exists, of a function of all the $x$ 's and the variable $y$, and therefore, when we replace $y$ by $g(x)$, gives the total differential of such a function with respect to the $x$ 's alone, along the curve $y=g(x)$. Applying this operator to the identity (1), therefore, we get a relation which is true at the point $a$, that is $\left(a_{1}, a_{2}, \ldots, a_{n}\right)$, the argument given in $\$ 8$ applying now, when we recall that the product and the sum of functions having an $n$-th differential at a point is a function having at that point an $n$-th differential. Hence the function $g(x)$, or $y$, bas a second differential at the point $a$, and it is given by the symbolic equation

$$
\left(h \frac{d}{d x}+\Delta y \frac{d}{d y}\right)\left(h \frac{d}{d x}+\Delta y \frac{d}{d y}\right) f=0,
$$

that is, by what was pointed out about the effect of the operator on $\Delta y$,

$$
\left(h \frac{d}{d x}\right)^{2} f+2 \Delta y\left(\frac{d}{d x}\right)\left(\frac{d}{d y}\right) f+(\Delta y)^{2}\left(\frac{d}{d y}\right)^{2} f+\frac{d f}{d y} \Delta^{2} y=0,
$$

where

$$
\begin{aligned}
\left(h \frac{d}{d x}\right)^{2} f & =\left(h_{1} \frac{d}{d x_{1}}+\ldots+h_{n} \frac{d}{d x^{\prime \prime}}\right)^{2} f \\
& =h_{1}^{2} f_{a_{1} a_{1}}+\ldots+h_{n}^{2} f_{a_{n} a_{n}}+2 h_{1} h_{2} f_{a_{1} a_{2}}+\ldots \\
& =D^{2} f
\end{aligned}
$$

the second differential of $f$ when $y$ is constant, and, similarly,

$$
\frac{d}{d x} \frac{d}{d y} f=D f_{y} .
$$

(Here it is to be remarked that we have identified mixed differential coefficients which oniy differ in the order of differentiation, which is correct, by the fundamental theorem in "The Theory of Differentials.")

$$
\text { Thus the equation } \quad\left(h \frac{d}{d x}+\Delta y \frac{d}{d y}\right)^{2} f=0
$$

may be written $\quad D^{2} f+2 \Delta y D f_{y}+(\Delta y)^{2} f_{y y}+f_{y} \Delta^{2} y=0$,

$$
\text { or } \quad\left(D^{2} f, D f_{y}, f_{y y}\right)(1, \Delta y)^{2}+f_{y} \Delta^{2} y=0 \text {, }
$$

a form which corresponds precisely to (ii.) of $\S 9$. 
Similarly, when $n=3$, we have for the third differential of $y$ at the point $a$, the symbolic equation

$$
\left(h \frac{d}{d x}+\Delta y \frac{d}{d y}\right)^{3} f=0
$$

or, which is the same thing,

$$
\left(D^{8} f, D^{2} f_{y}, D f_{y y}, f_{y y y} \backslash 1, \Delta y\right)^{3}+3\left(D f_{y}+\Delta y f_{y y}\right) \Delta^{2} y+f_{y} \Delta^{3} y=0 .
$$

It is unnecessary to dwell longer on these equations. Summing up we may say the $r$-th differential of $y$ at the point a is given symbolically by the equation

$$
\left(h \frac{d}{d x}+\Delta y \frac{d}{d y}\right)^{r} f=0
$$

just as, in the simple case when there is only one $x$, it was given by

$$
\left(\frac{d}{d y}+y^{\prime} \frac{d}{d y}\right)^{r} f=0
$$

This equation when expanded has precisely the same form as before, the successive differential coefficients of $y$ being replaced by the successive differentials $\Delta y, \Delta^{2} y, \Delta^{3} y, \ldots, \Delta^{v} y$, and differentiation with respect to $r$ being replaced by the operator $D$, which gives the partial differential of a function of the $x$ 's and $y$ with respect to the $x$ 's alone.

12. It should be noticed that in Theorem $5, \S 6$, although the uniqueness of the solution is involved in the proof of the continuity of $y$, considered as a function of $x$ in the neighbourhood of the point $x=a$, each such solution is continuous for any value of $x$ such that on the corresponding ordinate there is only one zero of $f(x, y)$, the neighbourhood being chosen sufficiently small; in particular, this is the case at the point $x=a$ itself, if there is no sequence of values of $y$ with $b$ as limit such that for each $f(a, y)=0$. This follows at once from the reasoning used in the proof of $\$ 6$. If this be the case, the reasoning used in the proof of the property (5) still applies whether or no the solution is unique; that is to say, if $f(x, y)$ has a first differential at $(a, b)$, each of the solutions has a differential coefficient at the point $x=a$, and the value of this differential coefficient is the same for all solutions and is given by

$$
f_{a}+f_{b} \frac{d y}{d x}=0 .
$$


An interesting application of this is constituted by the following theorem.

13. TheOREM 7.-If $g(x)$ is a function of $x$ which has the value $b$ when $x=a$, and possesses at the point $x=a$ a finite non-vanishing differential coefficient, then there exists at least one function $x$ of $y$ which has the value a when $y=b$, and in a certain closed neighbourhood of the point $y=b$ renders the equation

$$
y=g(x)
$$

an identity; further, all these functions possess at the point $y=b$ a common differential coefficient whose value is $1 / g^{\prime}(\alpha)$.

To prove this, we have, in fact, only to put

$$
f(x, y)=y-g(x),
$$

and apply the above. For, when $x=a$, there is no value of $y$ other than $b$ which makes $f(x, y)$ vanish.

14. It now follows at once from Theorem 5 that if the function $g(x)$, mentioned in the enunciation of the preceding theorem is in a closed neighbourhood of the point $x=a$, a monotone function of $x$, the function $x$ of $y$ is unique. This is in particular the cese if $g^{\prime}(x)$ exists in the neighbourhood of $x=a$, and is nowhere zero. (Theorem 5, Cor. 1.)

Applying Theorem $6, \S 7$, we get the following theorem, involving a most important case of uniqueness of the function $x$ of $y$.

Theorem 8.-If $g(x)$ is a function of $x$ which has the value $b$ when $x=a$, and possesses at the point $x=a$ a finite $n$-th differential coefficient where $n \geqslant 2$, and if $g^{\prime}(a)$ is different from zero, there is one, and only one, function $x$ of $y$ which has the value a when $y=b$, and renders the equation

$$
y=f(x)
$$

an identity in a certain closed neighbourhood of the point $y=b$. 


\section{J.IRT III.}

$\$ \$ 14-16$. Eristence and Differentials of Implicit Functions defined by Tico or More Equations.

14. 'Theorex 9.-If' $f_{1}(x, y), f_{2}(x, y), \ldots, f(x, y)$ are $r$ functions of the. $m$ variables $x$ and the r variables $y$, which are zero when the $x$ 's are equal to $a$ 's and the y's to b's, i.e., at the point $(a, b)$, and have n-th differentials there, where $n \geqslant 2$, and if the Jacobian $J$ of the f's with respect to the $y^{\prime}$,

$$
\left|\begin{array}{ccc}
\frac{d f_{1}}{d y_{1}}, & \ldots, & \frac{d f_{r}}{d y_{1}} \\
\frac{d f_{1}}{d y_{2}}, & \ldots, & \frac{d f_{r}}{d y_{2}} \\
\ldots & \ldots & \ldots \\
\frac{d f_{1}}{d y_{1}}, & \ldots, & \frac{d f_{r}}{d y_{r}}
\end{array}\right|
$$

is not zero at the point $(a, b)$, then there exist unique functions $y_{1}, y_{2}, \ldots, y$. of the mariables $x$, which hare the values $b_{1}, b_{2}, \ldots, b_{1}$. at the point $a$, and, throughout a closed neighbourhood of that point, make all the f's identically zero. Moreover these functions y have $n$-th differentials at the point a, whose values may be obtained from equations, which in symbolic form are

$$
\left(h \frac{d}{d x}+\Delta y \frac{d}{d y}\right)^{i} f_{j}=0 \quad(i=1,2, \ldots, n ; j=1,2, \ldots, r) .
$$

Since the Jacobian $J$ is not zero, all its principal minors are not zero, and we may assume, without loss of generality, that it has been so arranged that the leading principal minor is not zero. It then follows that one of the principal minors of that principal minor is not zero, and we may assume that the determinant is so arranged that that principal minor is the leading minor. Proceeding thus, we may assume that the determinant has been so arranged that none of the leading minors,

$$
J_{1}=\frac{d f_{1}}{d y_{1}}, \quad J_{2}=\left|\begin{array}{ll}
\frac{d f_{1}}{d y_{1}}, & \frac{d f_{2}}{d y_{1}} \\
\frac{d f_{1}}{d y_{1}}, & \frac{d f_{2}}{d ! y_{2}}
\end{array}\right|, \quad J_{3}=\left|\begin{array}{lll}
\frac{d f_{1}}{d y_{1}}, & \frac{d f_{2}}{d y_{1}}, & \frac{d f_{3}}{d y_{1}} \\
\frac{d f_{1}}{d y_{2}}, & \frac{d f_{2}}{d y_{2}}, & \frac{d f_{3}}{d y_{2}} \\
\frac{d f_{1}}{d y_{3}}, & \frac{d f_{2}}{d y_{3}}, & \frac{d f_{3}}{d y_{3}}
\end{array}\right| \ldots
$$


are zero at the point $(a, b)$. Then, since

$$
\frac{d f_{1}}{d b_{1}} \neq 0
$$

and $f_{1}$ has an $n$-th differential at the point $(a, b)$, where $n \geqslant 2$, there is, by Theorem 6 , one, and only one, function $y_{1}$ of the remaining $(m+r-1)$ variables $x$ and $y$ which has the value $b_{1}$ at the point $\left(a_{1}, \ldots, a_{m}, b_{2}, \ldots, b_{r}\right)$, and, in a certain closed neighbourhood of that point makes $f_{1}$ identically zero. Moreover, this function $y_{1}$ has an $n$-th differential at the point in question.

Now, replace $y_{1}$ in $f_{2}$ by the function so found, and call the result $F_{2}$. This is a function of the $m$ variables $x$ and the remaining $(r-1)$ rariables $y$, which has the value zero at the point $\left(a_{1}, \ldots, a_{m}, b_{2}, \ldots, b_{r}\right)$, since $y_{1}=b_{1}$ there.

Also, by Theorem 2 , it has an $n$-th differential at the same point since $f_{1}$ and $y_{1}$ both have $n$-th differentials. Also $d F_{2} / d y_{2}$ is not zero at the point, since, $F_{2}$ having a differential with respect to the $x$ 's and $y$ s, and therefore with respect to the y's, at the point and in its neighbourhood.

where

$$
\frac{d F_{2}}{d y_{2}}=\frac{d \dot{f}_{2}}{d y_{2}}+\frac{d f_{2}}{d y_{1}} \frac{d y_{1}}{d y_{2}}
$$

$$
\frac{d f_{1}}{d y_{2}}+\frac{d \dot{f}_{1}}{d y_{1}} \frac{d y_{1}}{d y_{2}}=0 .
$$

so that

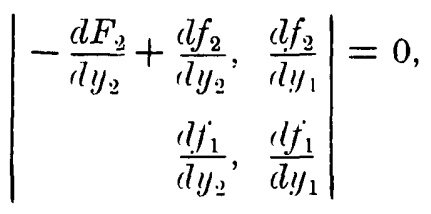

or

$$
J_{1} \frac{d F_{2}}{d y_{2}}+J_{2}=0
$$

Since $J_{1}^{2}$ and $J_{2}$ are both different from zero at the point, this grives a finite value different from zero for $d F_{2,} r l b_{2}$.

We can therefore again apply Theorem 6 , and assert that one, and only one, function $y_{2}$ of the $m$ variables $x$ ind the remaining $(r-2)$ variables $y$ exists, which has the value $b_{2}$ at the point $\left(a_{1}, \ldots, a_{m} . b_{3}, \ldots . l_{r}\right)$, and in a certain closed neighbourhood or that point makes $F_{2}$ illentically zero. Moreover this function $y_{2}$ has an $n$-th differential at the point.

SER. 2. VOL. T. No. 1 US1. 
Now, the effect of inserting this function $y_{2}$ in $F_{2}$ is obviously the same as that of inserting this $y_{2}$ in the $y_{1}$ previously found, and then in $f_{2}$ inserting this last $y_{1}$, and the function $y_{2}$ wherever it occurs explicitly Doing this both in $f_{1}$ and in $f_{2}$ they become functions of the $(m+r-2)$ variables which vanish identically. Making the same change in $f_{3}$ and denoting the result by $F_{3}$, we thus get the three equations

$$
\begin{aligned}
\frac{d F_{3}}{d y_{3}} & =\frac{d f_{3}}{d y_{3}}+\frac{d f_{3}}{d y_{2}} \frac{d y_{2}}{d y_{3}}+\frac{d f_{3}}{d y_{1}} \frac{d y_{1}}{d l_{3}}, \\
0 & =\frac{d f_{2}}{d y_{3}}+\frac{d f_{2}}{d y_{2}} \frac{d y_{2}}{d y_{3}}+\frac{d f_{2}}{d ! y_{1}} \frac{d y_{1}}{d y_{3}}, \\
0 & =\frac{d f_{1}}{d y_{3}}+\frac{d f_{1}}{d y_{2}} \frac{d y_{2}}{d y_{3}}+\frac{d f_{1}}{d y_{1}} \frac{d y_{1}}{d y_{3}}
\end{aligned}
$$

holding at the point in question, whence

$$
J_{2} \frac{d F_{9}}{d ! \eta}+J_{3}=0 .
$$

Thus $d F_{3} / d y_{3}$ is finite and different from zero at the point $\left(a_{1}, \ldots, a_{n}, b_{4}, \ldots, b_{r}\right)$, at which, as before, $F_{3}$ vanishes, and has an $n$-th differential. Thus we can again apply the theorem and deduce the existence of one, and only one, function $y_{3}$ of the $(m+r-3)$ variables having the value $b_{3}$ at the point, and in a certain closed neighbourhood of it making $F_{3}$ identically zero. This process may be continued and it is evident that we shall at each stage obtain unique functions $y_{1}, y_{2}, \ldots, y_{i}$, of the remaining $(m+r-i)$ variables having the proper values at the point considered, and having $n$-th differentials there, and making $f_{1}, f_{2}, \ldots, f_{g}$ vanish identically in a certain neighbourhood of the point. Inserting these values in $f_{i+1}$ and denoting the result by $F_{i+1}$, we then clearly get the equations

$$
\begin{aligned}
\frac{d F_{i+1}}{d ! y_{i+1}} & =\frac{d f_{i+1}}{d ! y_{i+1}}+\frac{d f_{i+1}}{d ! y_{i}} \frac{d y_{i}}{d y_{i+1}}+\ldots+\frac{d f_{i+1}}{d y_{1}} \frac{d y_{1}}{d y_{i+1}}, \\
0 & =\frac{d f_{i}}{d ! y_{i+1}}+\frac{d f_{i}}{d ! y_{i}} \frac{d y_{i}}{d y_{i+1}}+\ldots+\frac{d f_{i}}{d y_{1}} \frac{d y_{1}}{d y_{i+1}}, \ldots
\end{aligned}
$$

holding at the point, so that

$$
J_{i} \frac{d F_{i+1}}{d y_{i+1}}+J_{i+1}=0 .
$$

Thus again we can apply the theorem and proceed a stage further. This 
may be continued until we have exhausted all the $y$-coordinates, when we shall have expressed each of them in one, and only in one, way, so as to have the values $b$ at the point $a$, and, in a certain closed neighbourhood of that point, to make the $r$ functions $f$ vanish identically; moreover, these functions $y$ of the $x$ 's have $n$-th differentials at the point. This being so we have only to form the total differentials of the functions $f$ with respect to the $x$ 's, regarding the $y$ 's as being these functions, and equate the result to zero, to obtain equations which determine the values of the differentials of the $y$ 's at the point $a$; these equations may be written, symbolically, in the form

$$
\left(h \frac{d}{d x}+\Delta y \frac{d}{d y}\right)^{i} f_{j}=0 \quad(i=1,2, \ldots, n ; j=1,2, \ldots, r)
$$

Theorem 10.- If $f_{1}(x, y), f_{2}(x, y), \ldots, f_{r}(x, y)$ are $r$ functions of the $m$ variables $x$ and the $r$ variables $y$ which are zero at the point $(a, b)$, and have first differentials at the point $(a, b)$ and in a closed neighbourhood of that point first differentials with respect to the y's, and if in that closed neighbourhood the Jacobian $J$ of the f's with respect to the $y$ 's is not zero, and the same is true of one of its principal minors $J_{r-1}$, and of one of the principal minors $J_{r-2}$ of $J_{r-1}$, and so on, down to one of the common constituents of $J, J_{r-1}, \ldots, J_{2}$, then there exist unique functions $y_{1}, y_{2}, \ldots, y_{r}$ of the $m$ variables $x$, which have the values $b_{1}, b_{2}, \ldots, b$. at the point a, and, throughout a closed neighbourhood of that point, malie all the f's identically zero. Moreover, these functions !f have first differentials at the point a, whose values may be obtained by solving the equations

$$
\left(h \frac{l}{d x}+\Delta y \frac{d}{d y}\right) f_{i}=0
$$

that is, $h_{1} \frac{d f_{i}}{d x_{1}}+h_{2} \frac{d f_{i}}{d x_{2}}+\ldots+h_{m} \frac{d f_{i}}{d x_{n}}+\Delta y_{1} \frac{d f_{i}}{d y_{1}}+\ldots+\Delta y_{r} \frac{d l_{i}}{\left(! y_{r}\right.}=0$ for all integers i from 1 to $r$ both inclusive.

The proof of this theorem is essentially the same as that of the preceding theorem, quoting 'Theorem 5, Cor. 1 , instead of 'Theorem 6.

Con. 1.-The conditions that $J, J_{r-1}, \ldots, J_{2}, J_{1}$ should not ranish in a closed neighbourhood of the point $(a, b)$, being replacel by the cunditions that they should not vanish at the point and be continuous there. the theorem still holds. 
CoR. 2.-The same conditions being replaced by the conditions that $J$ should not vanish at the point, and all the partial differential coefficients of the f's with respect to the y's should be continuous at the point, the theorem still holds.

Con. 3.-The conditions that the f's should have first differentials with respect to the y's in a closed neighbourhood of the point, and that $J, J_{r-1}, \ldots, J_{1}$ should not vanish throughout that neighbourhood, may be replaced by the conditions that all the partial differential coefficients of the f's with respect to the y's should be continuous throughout a closed neighbourhood of the point, and the Jacobian J should not be zero at the point, the theorem then still holds.

A particular case of this is a theorem due to Dini that, if $J$ does not vanish at the point $(a, b)$, and all the partial differential coefficients of the $f$ 's are continuous, there is a unique set of solutions $y_{1}, \ldots, y_{r}$, each of which then will have a first differential at the point.

16. If in the preceding theorem we only assume that at the point $(a, b)$, but not necessarily in the neighbourhood, $J, J_{r-1}, \ldots$ are not zero, it is evident that we still get at least one set of solutions, the uniqueness of this set being the only thing affected. An argument similar to that given in $\$ 12$ shews that each such solution is continuous for any ensemble $x$, such that there is only one ensemble $y$ for which the given functions $f_{1}, f_{2}, \ldots, f_{r}$ all vanish. In particular this will be the case at the point $x=a$, provided the point $y=b$ is not a limiting point of points $y$ for which $f_{1}(a, y), f_{2}(a, y), \ldots, f_{r}(a, y)$ all vanish.

Supposing this to be the case, the reasoning by which the existence of the first differentials was demonstrated still holds, and the equations determining them are the same for all possible sets of solutions.

We thus easily get the following theorem, which corresponds to Theorem 7.

TheOREM 11.-If $g_{1}(x), g_{2}(x), \ldots, g_{r}(x)$ are functions of the $r$ variables $x$ which have the values $b_{1}, b_{2}, \ldots, b_{r}$ when the $x$ 's have the values $a_{1}, \ldots, a_{r}$, and possess at the point $x=a$ a finite non-vanishing Jacobian $J$, which has a non-vanishing principal minor $J_{r-1}$, and so on, down to $J_{1}$, then there exists at least one set of functions $x$ of the $r$ variables $y$ which 
have the values $a_{1}, \ldots, a_{r}$ at the point $y=b$, and which in a certain closed neighbourhood of that point render the equations

$$
y_{1}=f_{1}(x), \quad y_{2}=f_{2}(x), \ldots, y_{r}=f_{r}(x),
$$

identities; further, all these sets of functions possess at the point $y=b$ a common Jacobian $J^{\prime}$ whose value is $1 / J$.

Moreover, corresponding to Theorem 8, we may assert the uniqueness of the set of solutions, provided the $f$ 's have $n$-th differentials, where $n \geqslant 2$, and it will follow that the solutions themselves possess $n$-th differentials at the point $x=a$.

[I take this opportunity of pointing out an oversight in one of my previous papers, viz., that on "Oscillating Successions of Continuous Functions," pp. 303, 304. The set of points in Cor. 2 is only an ordinary inner limiting set when certain of the points at which the upper functions $=k$ are added to it. A similar remark applies to the lower function, and to the statement regarding the measure of the oscillation.

I may add that this correction does not affect the validity of the reasoning of my paper on "Trigonometrical Series," Mess. MIath., 1908, pp. 44,48 , though it necessitates a slight modification in the wording of the proof.] 